\title{
Healthcare costs and utilization for privately insured patients treated for non-infectious uveitis in the USA
}

David S Chu ${ }^{1,2^{*}}$, Scott J Johnson ${ }^{3}$, Usha G Mallya ${ }^{4}$, Matthew R Davis ${ }^{3}$, Rachael A Sorg ${ }^{3}$ and Mei Sheng Duh ${ }^{3}$

\begin{abstract}
Background: The purpose of this study was to describe comorbidities, healthcare costs, and resource utilization among patients with chronic non-infectious uveitis initiating corticosteroid, immunosuppressants, or biologics. In this retrospective cohort study, patients with a non-infectious uveitis diagnosis and continuous insurance coverage during a 6-month baseline were selected from a privately insured claims database with 80.7 million enrollees. Index dates were defined as the first prescription/administration of a corticosteroid, immunosuppressant, or biologic between 2003 and 2009. Comorbidities, healthcare costs, and utilization were analyzed in a per-member-per-month (PMPM) framework to account for varying between-patient treatment periods, defined as continuous medication use within the same class. Wilcoxon rank-sum and chi-square tests were used for comparisons of costs and categorical outcomes.
\end{abstract}

Results: Patients on corticosteroids $(N=4,568)$, immunosuppressants $(N=5,466)$, and biologics $(N=1,694)$ formed the study population. Baseline PMPM inpatient admission rates were 0.029 for patients on corticosteroids, 0.044 for patients on immunosuppressants, and 0.045 for patients on biologics ( $p<0.001$ immunosuppressants or biologics versus corticosteroids); during treatment, PMPM inpatient admissions increased to 0.044 and 0.048 for patients taking corticosteroids and immunosuppressants, respectively, but decreased to 0.024 for patients taking biologics ( $p<0.001$ versus corticosteroids and $p=0.003$ versus immunosuppressants). Baseline average PMPM costs for patients taking corticosteroids, immunosuppressants, and biologics were US\$935, US\$1,738, and US $\$ 1,439$ ( $p<0.001$ between groups), while on-treatment PMPM costs excluding drug costs increased to US\$1,129 for patients taking corticosteroids but lowered to US\$1,592 for patients taking immunosuppressants, and US\$918 for patients taking biologics ( $p<0.001$ versus corticosteroids or immunosuppressants).

Conclusions: There is significant economic burden associated with existing treatments of uveitis. Corticosteroids may be overused as a treatment for uveitis.

Keywords: Comorbidities; Healthcare costs; Healthcare utilization; Uveitis

\section{Background}

Uveitis is an inflammatory condition of the eye that is typically characterized by redness, pain, light sensitivity, and blurred/decreased vision and is associated with numerous ocular diseases and systemic conditions. This inflammation can also be categorized by the location of inflammation, including anterior uveitis

\footnotetext{
*Correspondence: chuda@njms.rutgers.edu

'Institute of Ophthalmology and Visual Science, New Jersey Medical School, Rutgers University, 90 Bergen Street, Suite 6100, Newark, NJ 07960, USA

${ }^{2}$ Metropolitan Eye Research and Surgery Institute, Palisades Park, NJ, USA Full list of author information is available at the end of the article
}

(e.g., iritis), intermediate uveitis (e.g., pars planitis, vitritis), and posterior uveitis (e.g., choroiditis, retinitis). Anterior uveitis is the most common form of uveitis in most populations, particularly in Western countries, accounting for about $50 \%$ to $60 \%$ of all uveitis cases in most tertiary referral centers and around $90 \%$ in primary care settings [1]. The majority of uveitis-related visual morbidity occurs in patients with posterior segment uveitis, which includes intermediate, posterior, and panuveitis.

Uveitis is responsible for an estimated $10 \%$ of cases of blindness in the USA [2,3], including 30,000 new cases 
of legal blindness each year [4]. Uveitis is a major cause of visual morbidity in the working age group [5]. Early diagnosis and treatment are important to prevent the vision-threatening complications of uveitis, including cataract, glaucoma, retinopathy, and macular edema [6]. Goals of treatment include suppressing inflammation and achieving remission [7].

Three drug classes that constitute the primary treatment modalities for uveitis include corticosteroids, traditional (non-biologic) immunosuppressive agents, and biologics. Corticosteroids are typically used as the first-line drug therapy for non-infectious inflammatory conditions [8]. They may be used topically, administered systemically via oral, intravenous or intramuscular route, or injected periocularly or implanted surgically. Serious side effects such as hypertension, cardiac failure, weight gain, osteoporosis, myopathy, osteonecrosis, and gastrointestinal side effects [9] are associated with the chronic use of systemic corticosteroids. If a patient's uveitis is not completely quiet after several weeks of high-dose corticosteroids and maintained with $10 \% \mathrm{mg}$ per day of prednisone (or equivalent) within $3 \%$ months or if the posterior segment is being affected, established guidelines recommend the use of steroid-sparing agents [10], such as second-line traditional immunosuppressant therapy (i.e., antimetabolites, $\mathrm{T}$ cell inhibitors and alkylating agents) $[8,11]$.

For patients whose uveitis condition is refractory to traditional immunosuppressants, biologic therapies may be considered. Examples of biologics used as a thirdline therapy include tumor necrosis factor (TNF) inhibitor agents, such as infliximab and adalimumab; interferons, such as recombinant human IFN- $\alpha-2 \mathrm{a}$ and IFN- $\alpha-2 b$; and anti-interleukin therapy [12]. Even though there have been a large number of reports on the use of biologic therapies to treat uveitis, there have been no controlled trials comparing the efficacy of different biologic therapies with each other or with traditional immunosuppressants, and further research is needed to support clinical decisions regarding choice of agent, time of initiation, and course of therapy [12].

Claims data analyses provide large samples for empirical analyses, which could contribute to understanding the economic burden related to uveitis as well as indicators for ophthalmologic comorbidities. Previous research has examined the incidence and prevalence of uveitis in a privately insured population in Northern California and Medicare populations [13,14]. Gritz and Wong [13] found that the incidence of uveitis was higher than results from smaller previous studies, the prevalence increased with age, and women had a higher prevalence of uveitis than men. Reeves et al. [14] found that the burden of uveitis is higher in an elderly population than previous research had indicated.
In comparison to previous studies, the current study utilizes pharmacy and non-elderly claims excluded in the analysis by Reeves et al. [14] and includes a larger sample to describe disease burden related to and treatment patterns for patients receiving treatment for uveitis. Specifically, we examine baseline characteristics, uveitisrelated ophthalmologic outcomes, healthcare utilization, and healthcare costs among patients receiving the three treatment options (i.e., corticosteroids, immunosuppressive agents, and biologics) with non-infectious uveitis in a privately insured population. We use a large, longitudinal claims data as an efficient way to study uncommon diseases like non-infectious uveitis [15].

\section{Methods}

\section{Data and study design}

The Thomson Reuters MarketScan ${ }^{\circledR}$ Commercial Databases, which have been used previously to estimate the economic burden of ophthalmologic disease [16], was used in this analysis. This database contains individual-level claims and enrollment data for approximately 80.7 million members from January 2003 to October 2009. The data include the enrollment history, demographics, medical claims, and pharmacy claims for employees, dependents, and retirees in the USA with primary insurance coverage.

We used a retrospective cohort design to address our research questions. Retrospective cohorts using claims data can provide equally valid results as a prospective cohort study while being more economical [17].

\section{Patient selection}

Prior literature was used to identify codes for noninfectious uveitis using the International Classification of Diseases, Ninth Revision, Clinical Modification (ICD-9$\mathrm{CM}$ ) coding system. Diagnosis codes including both posterior and anterior disease were used in the base case (Additional file 1: Table S1 for a complete list of ICD-9-CM codes). In a sensitivity analysis, codes more specific to posterior disease were used.

Patients were included in the sample if they had at least one claim for non-infectious uveitis diagnosed by an ophthalmologist (or optometrist) or two claims diagnosed by other physician specialists. Patients were also required to have at least one prescription dispensing or administration of corticosteroids, immunosuppressants, or biologics on or after their diagnosis of non-infectious uveitis (the list of drugs included can be found in Additional file 1: Table S2). In order to exclude patients with post-surgical inflammation, patients with incisional intraocular surgery within 3\% months of their uveitis diagnosis were excluded.

Patients were also required to have at least $6 \%$ months of continuous insurance coverage prior to their first prescription or administration, which was considered their baseline period, as well as at least $10 \%$ days of 
study period drug use. In order to identify patients with chronic uveitis, patients receiving corticosteroids had to have continuous prescriptions for 60 or more days. Sensitivity analyses requiring 90 or 30 or more days were implemented. Patients over 65\% years of age were excluded from the analysis to avoid incomplete claims due to Medicare dual coverage. Figure 1 contains additional detail on patient selection.

Three samples were developed, including patients receiving their first dose of corticosteroid therapy only (CTS), traditional immunosuppressants/immunomodulators (IMS) with or without corticosteroids, and biologics (BIO) with or without corticosteroids or immunosuppressants. Patients' first date of therapy was considered their index date. Additionally, patients were required to have no evidence of their sample's respective drug therapy in the $6 \%$ months prior to their index date.
Patient gender, age, health insurance type, and index year were reported. To ascertain general comorbidity burden of the patients at baseline, we used the Deyo adaptation of the Charlson comorbidity index, excluding ophthalmologic diagnosis codes [18].

\section{Treatment periods}

Analyses were performed based on treatment periods, which form the study period in the analysis. Treatment periods were defined as the continuous on-therapy period for each patient within the same class, spanning from a patient's index date to the date of either stopping therapy, stepping up therapy, or being censored in the data (e.g., disenrollment from health plan). Stopping therapy was based on the patient not refilling the prescription for a period of days equal to $150 \%$ the number of days in the previous prescription, based on the days supply variable in

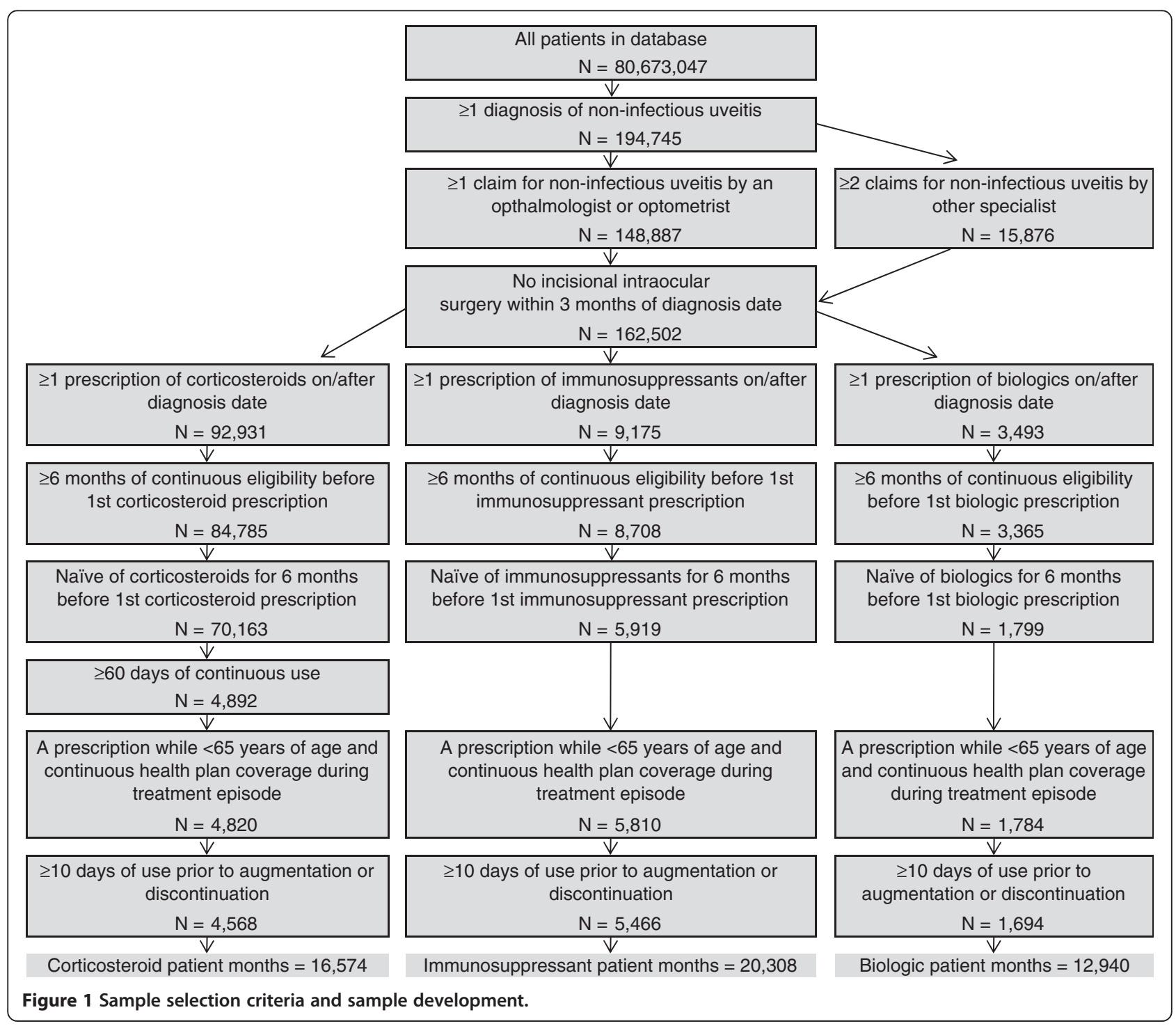


the pharmacy claims for prescription drugs [19] and based on the literature for physician-administered intramuscular injectables and infusions [12]. For example, if a patient received a first prescription of prednisone on 01 April 2007 with a $30 \%$ days' supply, then a second 30-day script on May 16 (which is a treatment gap of $15 \%$ days or $50 \%$ of the days' supply of the preceding script), and then no further prescriptions, a steroid episode of $30+$ $15+30 \%$ days would be assumed.

Stepping up therapy ended a treatment episode and occurred on the day that a patient received a prescription or administration for a higher class of therapy (e.g., from corticosteroids to immunosuppressants or immunosuppressants to biologics). Switching within the same class (e.g., from one immunosuppressant agent to another) did not end a treatment episode.

\section{Outcomes}

Healthcare costs and utilization were measured in a per-member-per-month (PMPM) framework to standardize estimates across patients with treatment periods of different lengths. Fractional months were grossed up to complete months based on a patient's observed treatment days.

Healthcare costs were measured using the paid amount by insurers for patient's covered benefits. Categories of costs include costs related to inpatient care, emergency room (ER) care, office or outpatient (OP) care, and care for each type of therapy. Costs were inflation adjusted to 2009 dollars based on the medical consumer pricing index. Utilization was measured in terms of monthly inpatient stays per patient and inpatient days per hospitalized patient, number of ER visits, number of OP visits, and number of prescription drug scripts.

Kaplan-Meier analysis was used to assess time to augment or switch to a next-step class of therapy. Augmenting therapy was considered to be adding a new class of therapy before the current treatment episode had ended. Switching therapy was based on beginning a new class of therapy at any time observable in the data before a censoring event, including loss of eligibility or the last available data in September 2009.

Though not a primary focus of the analysis, ophthalmologic outcomes in terms of counts of diagnoses codes for retinal detachments (ICD-9-CM codes: 361.0-361.3, 361.8, 361.9), glaucoma (ICD-9-CM codes: 365.0-365.6, 365.8, 365.9), cataracts (ICD-9-CM codes: 366.0-366.5, $366.8,366.9$ ), cystoid macular degeneration (ICD-9-CM 362.53), visual disturbances (ICD-9-CM codes: 368.1, 368.3-368.6, 368.8, 368.9), blindness (ICD-9-CM codes: 369.0-369.4, 369.6-369.9), and other visual complications including phthisis bulbi, chorioretinal scars, other macular scars, optic atrophy, optic neuritis, other disorders of optic nerve, hypotony, and band-shaped keratopathy (ICD-9CM codes: 360.4, 363.3, 363.32, 377.1, 377.3, 377.4, 360.3, and 371.4) were also reported. Additional file 1: Table S3 contains additional detail.

\section{Sensitivity analysis}

Comparison of baseline and study period all-cause costs were made for the CTS, IMS, and BIO samples excluding patients with rheumatoid arthritis, Crohn's disease, psoriasis, or ankylosing spondylitis based on their ICD-9-CM diagnosis codes in the baseline or study period. Patients with these conditions may receive any of these treatments, so we excluded them in a sensitivity analysis.

Posterior uveitis may be more severe than anterior disease, though some codes may describe both types of disease. In a second sensitivity on all-cause costs, we include patients using a subset of ICD-9-CM codes that are more specific to posterior disease (see Additional file 1: Table S1). Lastly, we also assessed results excluding diagnoses made only by non-ophthalmologists in order to increase the accuracy of the non-infectious uveitis diagnosis.

\section{Statistical analysis}

All analyses were conducted using SAS version 9.2 (SAS Institute Inc., Cary, NC, USA). Statistical comparisons were conducted using chi-square tests for categorical outcomes and Wilcoxon rank-sum tests for continuous outcomes within baseline and study periods. Wilcoxon signed-rank tests assessed differences in pre- versus postcomparisons.

\section{Results}

The distribution of the study population was $38.9 \%$ CTS $(N=4,568), 46.6 \%$ IMS $(N=5,466)$, and $14.4 \%$ BIO $(N=1,694)$ out of 11,728 patients. The study periods were $16,574,20,308$, and 12,940 patient-months for the CTS, IMS, and BIO groups. Approximately $93 \%$ of the CTS were $(65,271$ of 70,163$)$ patients excluded from the CTS sample as a result of patients having fewer than 60 continuous days of corticosteroids prescriptions.

As shown in Table 1, about $84 \%$ (9,808 of 11,728 total patients) of patients were between age 35 and 64 at their index date. A higher proportion of women receive IMS (72.1\%, 3,940 of 5,466 IMS patients) than BIO (61.4\%, 1,040 of 1,694 BIO patients) or CTS $(59.7 \%, 2,729$ of 4,568 CTS patients) $(p<0.0001$ for IMS versus CTS or BIO). CTS patients were more likely to be enrolled in an HMO ( $p=0.0001$ for CTS vs. IMS; $p=0.0004$ for CTS versus $\mathrm{BIO})$. The numbers of patients diagnosed has grown over time, with 2009 reflecting only $9 \%$ months of available data. Between therapies, there were no apparent trends related to the year in which therapy was prescribed. In other words, neither IMS nor BIO therapy shows an increase in the rate of use for patients fitting the selection criteria over the period from 2003 to 2009. 
Table 1 Baseline demographics for non-infectious uveitis patients by treatment groups

\begin{tabular}{|c|c|c|c|}
\hline & $\begin{array}{l}\text { CTS patients }^{\mathrm{a}} \\
(N=4,568)\end{array}$ & $\begin{array}{l}\text { IMS patients }^{\mathrm{b}} \\
(N=5,466)\end{array}$ & $\begin{array}{c}\text { BIO patients } \\
(N=1,694)\end{array}$ \\
\hline \multicolumn{4}{|l|}{ Gender, N (\%) } \\
\hline Male & $1,839(40.3 \%)$ & $1,526(27.9 \%)^{a}$ & $654(38.6 \%)^{\mathrm{b}}$ \\
\hline Female & 2,729 (59.7\%) & $3,940(72.1 \%)^{\mathrm{a}}$ & $1,040(61.4 \%)^{b}$ \\
\hline \multicolumn{4}{|l|}{ Age, N (\%) } \\
\hline $0-17, \%$ & $162(3.5 \%)$ & $350(6.4 \%)^{\mathrm{a}}$ & $153(9.0 \%)^{a, b}$ \\
\hline $18-24$ & $142(3.1 \%)$ & $170(3.1 \%)^{\mathrm{a}}$ & $77(4.5 \%)^{a, b}$ \\
\hline $25-34$ & $305(6.7 \%)$ & $388(7.1 \%)^{a}$ & $173(10.2 \%)^{a, b}$ \\
\hline $35-44$ & 704 (15.4\%) & $868(15.9 \%)^{\mathrm{a}}$ & $390(23.0 \%)^{a, b}$ \\
\hline $45-54$ & $1,360(29.8 \%)$ & $1,683(30.8 \%)^{a}$ & $472(27.9 \%)^{a, b}$ \\
\hline $55-64$ & $1,895(41.5 \%)$ & $2,007(36.7 \%)^{\mathrm{a}}$ & $429(25.3 \%)^{a, b}$ \\
\hline \multicolumn{4}{|c|}{ Index year, $N(\%)$} \\
\hline 2003 & 175 (3.8\%) & $219(4.0 \%)$ & $72(4.3 \%)$ \\
\hline 2004 & $401(8.8 \%)$ & 507 (9.3\%) & $151(8.9 \%)$ \\
\hline 2005 & $641(14.0 \%)$ & $723(13.2 \%)$ & $208(12.3 \%)$ \\
\hline 2006 & $730(16.0 \%)$ & $803(14.7 \%)$ & $250(14.8 \%)$ \\
\hline 2007 & $862(18.9 \%)$ & 1,088 (19.9\%) & $346(20.4 \%)$ \\
\hline 2008 & 1,063 (23.3\%) & $1,280(23.4 \%)$ & $420(24.8 \%)$ \\
\hline 2009 & $696(15.2 \%)$ & $846(15.5 \%)$ & $247(14.6 \%)$ \\
\hline \multicolumn{4}{|c|}{ Health insurance type, $N(\%)$} \\
\hline $\mathrm{HMO}$ & $924(20.2 \%)$ & $924(16.9 \%)^{\mathrm{a}}$ & $282(16.6 \%)^{a}$ \\
\hline PPO & 2,456 (53.8\%) & $3,216(58.8 \%)^{a}$ & $1,014(59.9 \%)^{\mathrm{a}}$ \\
\hline EPO & $18(0.4 \%)$ & $34(0.6 \%)^{a}$ & $7(0.4 \%)^{a}$ \\
\hline Indemnity & $72(1.6 \%)$ & $94(1.7 \%)^{\mathrm{a}}$ & $29(1.7 \%)^{a}$ \\
\hline Other & $1,098(24.0 \%)$ & $1,198(21.9 \%)^{\mathrm{a}}$ & $362(21.4 \%)^{a}$ \\
\hline
\end{tabular}

$\mathrm{HMO}$, health maintenance organization; PPO, preferred provider organization; EPO, extended provider organization. Chi-square tests were used to test differences in categorical variables. ${ }^{\mathrm{a}, \mathrm{b}}$ Data with superscripted letter(s) indicate a significant difference $(P<0.05)$ from the uveitis subtype represented by that letter.

As shown in Table 2, in the $6 \%$ months prior to initiating therapy, BIO and IMS patients were associated with the highest Charlson comorbidity index score. IMS patients were associated with the highest level of glaucoma, cataracts, and visual disturbances. Both IMS and $\mathrm{BIO}$ patients had greater baseline rates of cystoid macular degeneration than did CTS patients.

Small proportions of CTS patients had evidence of prior IMS (6.1\%, 280 of 4,568 CTS patients) or BIO (2.5\%, 112 of 4,568 CTS patients) use before their index CTS dose, indicating that the sample was not completely naïve to therapy. Most IMS patients received steroids (69.2\%, 3,784 of 5,466 IMS patients), and some IMS patients had received biologics (5.7\%, 313 of 5,466 IMS patients). Most $\mathrm{BIO}$ patients had received steroids (71.8\%, 1,217 of 1,694 BIO patients), and more than one-third received IMS $(38.5 \%, 652$ of 1,694 BIO patients). These treatment patterns are consistent with step therapy, where fewer patients advance to higher levels of therapy.

During the baseline period, IMS patients were associated with the highest monthly all-cause costs (US $\$ 1,738$ per month) followed by BIO (US\$1,439) and CTS patients (US\$935) $(p<0.0001$ between each). Inpatient costs represented approximately $31.5 \%$ (US\$294 of US\$935), $39.8 \%$ (US\$692 of US\$1,738), and $28.4 \%$ (US $\$ 408$ of US $\$ 1,439)$ of total costs in the CTS, IMS, and BIO samples.

Monthly rates of hospitalization and emergency room visits in the $6 \%$ months prior to initiating therapy were significantly different among the therapy groups. In terms of patients who had any inpatient stay, BIO patients were associated with the highest proportion of patients hospitalized per month (2.3\%, 39 of 1,694 BIO patients), followed by IMS patients $(2.1 \%, 114$ of 5,466 IMS patients) and CTS patients (1.4\%, 64 of 4,568 CTS patients) $(p<0.0001$ for IMS and BIO versus CTS). BIO patients were associated with the highest proportion of patients going to the ER per month (2.7\%, 46 of 1,694 BIO patients) followed by IMS patients (2.4\%, 132 of 5,466 IMS patients) and CTS patients (2.0\%, 89 of 4,568 CTS patients).

Though not a primary focus of our analysis, ophthalmologic comorbidities per patient in the study period are presented in Table 3. CTS patients were associated with a higher level of diagnoses of glaucoma, cystoid macular degeneration, and retinal detachments compared to IMS or BIO. CTS patients were also associated with a higher level of visual disturbances compared to IMS. Incidence of claims among CTS patients increased in the study period relative to the baseline in glaucoma, cystoid macular degeneration, retinal detachments, and visual disturbances; however, incidence of claims among IMS and BIO patients decreased or remained about the same in every category except for glaucoma, where it increased for both.

In the study period, most CTS patients (72\%, 3,288 of 4,568 CTS patients) neither augmented nor switched to IMS or BIO, and most IMS patients $(82 \%, 4,505$ of 5,466 IMS patients) neither augmented nor switched to BIO.

Study period utilization and costs appear in Table 4. As shown in Table 2, CTS patients were associated with the lowest number of monthly ER visits and inpatient stays per patient in the $6 \%$ months prior to their index date. In the study period, IMS patients were had the highest number of inpatient stays per patient. BIO patients had the highest number of inpatient stays and ER visits at baseline. However, in the post-index period, BIO patients had the lowest number of inpatient stays or ER visits. The $\mathrm{BIO}$ group was the only group to have a reduction in both the number of inpatient stays and ER visits. Monthly inpatient stays decreased by $0.021(p=0.0033)$ stays per patient and ER visits decreased by $0.012(p=0.0031)$ 
Table 2 Baseline ophthalmologic comorbidities, utilization, costs, and prior therapy for patients with non-infectious uveitis by treatment group

\begin{tabular}{|c|c|c|c|}
\hline & $\begin{array}{c}\text { CTS patients }^{\mathrm{a}} \\
(N=4,568)\end{array}$ & $\begin{array}{c}\text { IMS patients }^{\mathrm{b}} \\
(N=5,466)\end{array}$ & $\begin{array}{c}\text { BIO patients } \\
(N=1,694)\end{array}$ \\
\hline Patient months & 27,408 & 32,796 & 10,164 \\
\hline \multicolumn{4}{|l|}{ Baseline monthly ophthalmologic comorbidities, mean (std) } \\
\hline Glaucoma & $0.041(0.15)$ & $0.045(0.16)$ & $0.028(0.13)^{a, b}$ \\
\hline Cataract & $0.022(0.09)$ & $0.024(0.10)$ & $0.016(0.08)^{a, b}$ \\
\hline Cystoid macular degeneration & $0.004(0.03)$ & $0.008(0.06)^{\mathrm{a}}$ & $0.008(0.07)^{\mathrm{a}}$ \\
\hline Retinal detachments & $0.006(0.05)$ & $0.007(0.06)$ & $0.005(0.06)^{a, b}$ \\
\hline Visual disturbances & $0.005(0.04)$ & $0.007(0.05)^{\mathrm{a}}$ & $0.006(0.05)$ \\
\hline Blindness & $0.001(0.02)$ & $0.002(0.02)^{a}$ & $0.002(0.02)^{\mathrm{a}}$ \\
\hline Other visual complications & $0.012(0.06)$ & $0.024(0.11)^{\mathrm{a}}$ & $0.019(0.11)^{b}$ \\
\hline Baseline Charlson Comorbidity Index (CCI), mean (std) & $0.482(1.06)$ & $0.730(1.29)^{\mathrm{a}}$ & $0.802(1.15)^{a, b}$ \\
\hline \multicolumn{4}{|l|}{ Baseline monthly comorbidities, mean (std) } \\
\hline Crohn's disease & $0.009(0.10)$ & $0.024(0.22)^{\mathrm{a}}$ & $0.086(0.43)^{a, b}$ \\
\hline Rheumatoid arthritis & $0.010(0.09)$ & $0.036(0.18)^{\mathrm{a}}$ & $0.125(0.33)^{a, b}$ \\
\hline Psoriasis & $0.005(0.10)$ & $0.017(0.18)^{a}$ & $0.054(0.31)^{\mathrm{a}, \mathrm{b}}$ \\
\hline Ankylosing spondylitis & $0.005(0.06)$ & $0.013(0.10)^{\mathrm{a}}$ & $0.095(0.23)^{a, b}$ \\
\hline Baseline monthly total healthcare costs, mean [median] (std) (US\$) & $935[279](2,840)$ & $1,738[492](5,763)^{a}$ & $1,439[597](3,171)^{\mathrm{a}, \mathrm{b}}$ \\
\hline Medical services & $735[134](2,741)$ & $1,497[288](5,668)^{a}$ & $1,204[375](3,039)^{a, b}$ \\
\hline Inpatient stay (IP) & $294[0](2,177)$ & $692[0](4,816)^{a}$ & $408[0](2,233)^{a}$ \\
\hline Emergency room (ER) & $18[0](119)$ & $24[0](142)^{a}$ & $26[0](113)^{a, b}$ \\
\hline Office/outpatient (OP) & $423[123](1,041)$ & $782[263](1,787)^{\mathrm{a}}$ & $771[337](1,528)^{a, b}$ \\
\hline Pharmacy services & $200[66](453)$ & $241[87](483)^{a}$ & $234[104](485)^{a, b}$ \\
\hline Corticosteroids & $0[0](0)$ & $3[0](9)^{a}$ & $3[0](11)^{a}$ \\
\hline Immunosuppressants & $9[0](82)$ & $0[0](0)^{a}$ & $20[0](104)^{a, b}$ \\
\hline Biologics & $24[0](193)$ & $48[0](271)^{\mathrm{a}}$ & $0[0](0)^{a, b}$ \\
\hline \multicolumn{4}{|l|}{ Baseline monthly total healthcare resource utilization } \\
\hline \multicolumn{4}{|l|}{ Inpatient stay (IP) } \\
\hline Number of patients, $N(\%)$ & $64(1.4 \%)$ & $114(2.1 \%)^{a}$ & $39(2.3 \%)^{\mathrm{a}}$ \\
\hline Number of stays/patient, mean (SD) & $0.029(0.19)$ & $0.044(0.22)^{\mathrm{a}}$ & $0.045(0.19)^{\mathrm{a}}$ \\
\hline Number of days/ hospitalized patient, mean (SD) & $3.88(8.05)$ & $4.133(5.76)$ & $3.908(4.03)^{\mathrm{a}}$ \\
\hline \multicolumn{4}{|l|}{ Emergency room (ER) } \\
\hline Number of patients, $N(\%)$ & $89(2.0 \%)$ & $132(2.4 \%)^{a}$ & $46(2.7 \%)^{a}$ \\
\hline Number of visits/patient, mean (SD) & $0.028(0.10)$ & $0.035(0.11)^{a}$ & $0.042(0.12)^{a}$ \\
\hline \multicolumn{4}{|l|}{ Office/outpatient (OP) } \\
\hline Number of patients, $N(\%)$ & $722(15.8 \%)$ & $900(16.5 \%)^{a}$ & $281(16.6 \%)^{a, b}$ \\
\hline Number of visits/patient, mean (std) & $1.426(1.48)$ & $2.172(1.82)^{a}$ & $2.372(1.82)^{a, b}$ \\
\hline \multicolumn{4}{|l|}{ Baseline treatment characteristics (over the 6 month period), $N(\%)$} \\
\hline Baseline any corticosteroid use & - & $3,784(69.2 \%)^{a}$ & $1,217(71.8 \%)^{a, b}$ \\
\hline Baseline any immunosuppressant use & $280(6.1 \%)$ & - & $652(38.5 \%)^{a, b}$ \\
\hline Baseline any biologic use & $112(2.5 \%)$ & $313(5.7 \%)^{\mathrm{a}}$ & - \\
\hline
\end{tabular}

CTS, corticosteroids; IMS, immunosuppressive therapy; BIO, biologics. Chi-square tests were used to test differences in categorical variables. Wilcoxon tests were used to test differences in continuous variables. ${ }^{\mathrm{a}, \mathrm{b}}$ Data with superscripted letter(s) indicate a significant difference $(P<0.05)$ from the uveitis subtype represented by that letter. 
Table 3 Study period ophthalmologic comorbidities and prior treatment for patients with non-infectious uveitis by treatment group

\begin{tabular}{|c|c|c|c|}
\hline & $\begin{array}{l}\text { CTS patients }^{\mathrm{a}} \\
(N=4,568)\end{array}$ & $\begin{array}{l}\text { IMS patients }^{\mathrm{b}} \\
(N=5,466)\end{array}$ & $\begin{array}{c}\text { BIO patients } \\
(N=1,694)\end{array}$ \\
\hline Patient months & 16,574 & 20,308 & 12,940 \\
\hline Mean months/treatment episode (time to discontinuation), mean (std) & $3.63(3.13)$ & $3.72(5.61)^{\mathrm{a}}$ & $7.64(9.36)^{a, b}$ \\
\hline \multicolumn{4}{|l|}{ Study period ophthalmologic comorbidities, mean (std) } \\
\hline Glaucoma & $0.058(0.22)$ & $0.052(0.22)^{a}$ & $0.034(0.14)^{a}$ \\
\hline Cataract & $0.022(0.12)$ & $0.025(0.11)$ & $0.016(0.07)$ \\
\hline Cystoid macular degeneration & $0.013(0.10)$ & $0.010(0.07)^{\mathrm{a}}$ & $0.005(0.04)^{a}$ \\
\hline Retinal detachments & $0.011(0.10)$ & $0.004(0.05)^{\mathrm{a}}$ & $0.002(0.03)^{a}$ \\
\hline Visual disturbances & $0.007(0.07)$ & $0.004(0.04)^{\mathrm{a}}$ & $0.003(0.02)$ \\
\hline Blindness & $0.001(0.03)$ & $0.002(0.03)$ & $0.000(0.01)$ \\
\hline Other visual complications & $0.011(0.09)$ & $0.015(0.12)$ & $0.007(0.05)$ \\
\hline \multicolumn{4}{|l|}{ Augmenting or switching } \\
\hline Augmented to immunosuppressants, N (\%) & $348(7.60 \%)$ & & \\
\hline Mean days to augmenting, conditional on augmenting, mean (std) & $57(56)$ & & \\
\hline Augmented to biologics, $N(\%)$ & $96(2.10 \%)$ & $346(6.30 \%)$ & \\
\hline Mean days to augmenting, conditional on augmenting, mean (std) & $42(37)$ & $78(116)^{a}$ & \\
\hline Switched to immunosuppressants, $N(\%)$ & $615(13.50 \%)$ & & \\
\hline Mean days to switching, conditional on switching, mean (std) & $213(299)$ & & \\
\hline Switched to biologics, $N(\%)$ & $221(4.80 \%)$ & $615(11.30 \%)$ & \\
\hline Mean days to switching, conditional on switching, mean (std) & $256(358)$ & $213(295)$ & \\
\hline
\end{tabular}

CTS, corticosteroids; IMS, immunosuppressive therapy; BIO, biologics. Chi-square tests were used to test differences in categorical variables. Wilcoxon tests were used to test differences in continuous variables. ${ }^{a, b}$ Data with superscripted letter(s) indicates a significant difference $(P<0.05)$ from the uveitis subtype represented by that letter.

visits per patient from baseline to the study period for the BIO group; however, inpatient stays increased by $0.015(p=0.0014)$ and $0.004(p=0.0007)$ stays per patient for the CTS and IMS groups. ER visits increased slightly by $0.002(p=0.0024)$ for CTS and decreased by 0.004 $(p<0.0001)$ for IMS.

In the study period, BIO patients were associated with the highest monthly total healthcare costs. While all-cause monthly healthcare costs during the study period increased considerably from baseline in the BIO group (+US\$1,250, $p<0.0001$ ) compared to the CTS $(+\mathrm{US} \$ 209, p<0.0001)$ and IMS (+US\$21, NS, $p=0.1075)$ groups, relatively sizable reductions in inpatient costs versus baseline occurred in the IMS (-US\$256, $p<0.0001$ ) and BIO groups (-US\$225, $p=0.0033$ ). All-cause monthly costs in the study period excluding study drugs were lowest in the BIO group. Differencing these costs versus the baseline resulted in cost increases in CTS (+US\$194, $p<0.0001)$ and decreases in IMS (-US\$146, $p<0.0001)$ and $\mathrm{BIO}(-\mathrm{US} \$ 521, p<0.0001)$.

In sensitivity analysis requiring $90(N=2,341)$ or 30 $(N=19,426)$ days or more of continuous corticosteroid use to be in the CTS sample, all-cause monthly costs excluding study drugs increased + US\$97 $(p=0.0002)$ and + US\$257 $(p<0.0001)$ in CTS.
When excluding patients with rheumatoid arthritis, Crohn's disease, psoriasis, or ankylosing spondylitis, baseline and study costs were generally consistent with the overall analysis. Average costs excluding study drugs in the CTS $(N=4,169)$ sample increased (+US $\$ 226, p<0.0001$ ); in the IMS $(N=4,287)$ sample, they increased slightly (+US $\$ 56, p<0.0001)$, and in BIO $(N=524)$, they decreased (-US\$841, NS, $p=0.3519$ ).

When using ICD-9-CM diagnosis codes more specific to posterior disease, average cost differences excluding study drugs between study and baseline periods increased $(+\mathrm{US} \$ 176, p<0.0001)$ in the CTS $(N=1,979)$ sample; increased (+US\$133, $p<0.0001)$ in the IMS $(N=2,465)$ sample, and decreased (-US\$768, $p=0.0152)$ in the BIO $(N=672)$ sample.

Lastly, when excluding patients who were not diagnosed by an ophthalmologist in order to increase the accuracy of the non-infectious uveitis diagnosis, baseline and study costs were also generally consistent with the overall analysis. Average costs excluding study drugs increased (+US\$228, $p<0.0001)$ in the CTS $(N=3,732)$ sample; decreased slightly (-US $\$ 178$, $p<0.0001)$ in the IMS $(N=4,144)$ sample; and decreased (-US\$567, $p<0.0001$ ) in the BIO sample $(N=1,323)$. 
Table 4 Study period utilization and costs for patients with non-infectious uveitis by treatment group

\begin{tabular}{|c|c|c|c|}
\hline & $\begin{array}{l}\text { CTS patients }^{\mathrm{a}} \\
(N=4,568)\end{array}$ & $\begin{array}{l}\text { IMS patients }^{b} \\
(N=5,466)\end{array}$ & $\begin{array}{l}\text { BIO patients } \\
(N=1,694)\end{array}$ \\
\hline \multicolumn{4}{|l|}{ Total monthly healthcare resource utilization per patient, mean (SD) } \\
\hline \multicolumn{4}{|l|}{ Inpatient stay (IP) } \\
\hline Number of stays/patient & $0.044(0.34)$ & $0.048(0.30)$ & $0.024(0.19)^{a, b}$ \\
\hline Number of days/ hospitalized patient & $3.678(3.89)$ & $3.621(4.18)$ & $3.862(3.68)^{b}$ \\
\hline \multicolumn{4}{|l|}{ Emergency room (ER) } \\
\hline Number of visits/patient & $0.030(0.13)$ & $0.030(0.13)$ & $0.030(0.13)^{a, b}$ \\
\hline \multicolumn{4}{|l|}{ Office/outpatient (OP) } \\
\hline Number of visits/patient & $2.025(1.81)$ & $2.365(1.95)$ & $1.939(1.55)$ \\
\hline \multicolumn{4}{|l|}{ Pharmacy services } \\
\hline Number of scripts/patient & $3.015(2.36)$ & $4.106(2.93)^{\mathrm{a}}$ & $3.389(2.66)^{\mathrm{a}, \mathrm{b}}$ \\
\hline Total monthly healthcare costs per patient, mean [median] (SD) (US\$) & $1,144[382](3,601)$ & $1,759[484](5,474)^{\mathrm{a}}$ & $2,689[2,194](2,912)^{a, b}$ \\
\hline Medical services (excluding study drugs) & $902[195](3,445)$ & $1,324[196](5,194)$ & $716[273](2,005)^{a, b}$ \\
\hline Inpatient stay (IP) & $307[0](2,497)$ & $436[0](3,891)$ & $183[0](1,444)^{a, b}$ \\
\hline Emergency room (ER) & $21[0](148)$ & $25[0](277)^{a}$ & $21[0](373)^{a, b}$ \\
\hline Office/outpatient (OP) & $574[185](1,720)$ & $863[186](2,482)$ & $512[252](1,110)^{a, b}$ \\
\hline Pharmacy services (excluding study drugs) & $227[82](576)$ & 268 [85] (538) & $201[87](398)$ \\
\hline Total study drug costs & 16 [7] (26) & $167[64](380)^{a}$ & $1,772[1,491](1,532)^{a, b}$ \\
\hline Total costs excluding study drug costs & $1,129[365](3,599)$ & $1,592[379](5,383)$ & $918[466](2,105)^{\mathrm{a}, \mathrm{b}}$ \\
\hline
\end{tabular}

CTS, corticosteroids; IMS, immunosuppressive therapy; BIO, biologics. Chi-square tests were used to test differences in categorical variables. Wilcoxon tests were used to test differences in continuous variables. Healthcare costs and visits were calculated per-member-per-month (PMPM). Sample means and standard deviations were weighted by treatment months. ${ }^{\mathrm{a}, \mathrm{b}}$ Data with superscripted letter(s) indicates a significant difference $(P<0.05)$ from the uveitis subtype represented by that letter.

\section{Discussion}

Little is known about the actual practice patterns of physicians treating uveitis. Our approach, using a retrospective analysis of a large claims data set, has advantages over previous research, which has generally relied on smaller samples that are often focused on the elderly, tertiary care centers, or a limited community population. Previous research has found that the types of cases seen in tertiary care centers or over limited geographies can vary based on disease etiology and anatomic location, limiting their generalizability [20]. Case series reports from subspecialty practices may not reflect what general ophthalmologists see and treat. The current study utilizes health insurance claims data from about one-quarter of the US population who are geographically diversely distributed and are cared for by hospital and community doctors alike, including inflammatory disorder ophthalmologists, general ophthalmologists, and other types of providers.

Findings indicate that the financial burden of chronic non-infectious uveitis is comparable to other medically and economically significant disease. Costs of treated patients with uveitis in the baseline and study periods were high for all groups relative to the average privately insured patient, whose average monthly (annual) spending in the USA was US\$323 (US\$3,868) in 2009 dollars [21]. Comparative study period costs per month in 2009 dollars for CTS, IMS, and BIO patients were US $\$ 1,144$, US $\$ 1,759$, and US $\$ 2,689$, respectively. After taking into account healthcare cost inflation, we estimated that the average cost for non-infectious uveitis patients ranges from 3.1 to 8.3 times the costs of the average privately insured patient in 2009. These observed mean monthly healthcare costs for non-infectious uveitis are similar or higher than those for diabetes or hypertension patients (US $\$ 1,016$ or US $\$ 723$ in 2009 dollars) [22] in a privately insured population and similar or lower than those for cancer patients (US\$2,649 for prostate cancer to US\$9,225 for pancreatic cancer in 2009 dollars) in a private and Medicare population [23].

In this study, a substantial proportion of chronic uveitis patients received CTS alone, and a substantial majority of these patients were never observed to switch to IMS or BIO. While the CTS may have resolved the uveitis for some patients, average costs, utilization and rates of ophthalmological comorbidities increased during the CTS treatment episodes, indicating evidence of increased disease burden. Similar findings were evident in the sensitivity analyses requiring 90 or $30 \%$ days of continuous corticosteroids to be included in the CTS sample; in the latter sensitivity, $N=19,426$ patients received CTS, $88 \%$ of whom had no evidence of receiving immunosuppressants or biologics later despite increased evidence of increased disease burden. 
Nguyen et al. [10] found that the majority of physicians that treat a high volume of uveitis cases (primarily ophthalmologists) preferred high CTS doses to treat non-infectious uveitis and did not adhere to currently recommended guidelines for management of uveitis. Guidelines recommend the addition of IMS as a steroid-sparing agent if inflammation cannot be controlled with $\leq 10 \mathrm{mg}$ /day of prednisone (or equivalent) within 3 months [8,24]. Additionally, our data showed no change in the treatment distribution of CTS versus IMS or BIO over 2003 to 2009, indicating that adoption of the guidelines was slow at best over this time period. Importantly, CTS therapy was associated with increases in hospital admissions and ER visits and all-cause costs. Continued use of CTS could be due to uncertainty regarding treating severe inflammation among community ophthalmologists, fear about side effects or skepticism about efficacy of IMS or $\mathrm{BIO}$, inexpensive drug costs of CTS, or lack of awareness of the current guidelines.

This research also provides evidence that IMS and BIO therapy may be more effective at reducing ophthalmologic comorbidities related to uveitis than CTS. Further, treatment with $\mathrm{BIO}$ was associated with the largest reductions in non-study drug (i.e., non-biologic) costs and declines in hospital admissions and ER visits. Most IMS (82\%) patients were never observed to switch to BIO in the data. Resistance to using biologics may be due to the lack of clinical trial evidence, the expense of the agents, perceptions about safety, and a lack of experience with using biologics.

Though not a focus of the study, we calculated simple measures of prevalence by counting the patients with uveitis based on diagnosis codes in 2003 to 2009, only in 2003, and only in 2008. Our simple prevalence estimate, in terms of patients who ever had a diagnosis of uveitis using the data, was about 201/100,000; using only those patients with diagnoses in 2003 and 2008, prevalence figures were 88 and 113/100,000. Gritz and Wong [13] estimated that their period prevalence, calculated as the mid-period prevalence in their one year study in Northern California, was 115.3 cases/100,000 persons. Reeves et al. [14] estimated that cumulative prevalence in a Medicare population of posterior uveitis ranged from 108 to 286/ 100,000 . Our estimates are generally lower but in the same range as Reeves et al., potentially because we excluded patients over age 65 due to dual insurance issues with private supplemental insurance or Medicare.

Limitations arise from the nature of the administrative claims data used in this study. Claims data are designed for billing purposes rather than research, and measurement error via miscoding of conditions may occur $[25,26]$. Further, lack of clinical detail in both disease etiology and severity may lump heterogeneous patient groups into the same disease category. Further research may consider risk adjusting patients at baseline, which was not performed in this analysis. Patients in our study treated with CTS are likely to vary significantly in the severity of their uveitis, and BIO patients had the highest average Charlson comorbidity index $(p=0.0001$ versus both). Patients receiving both corticosteroids and immunosuppressants are included in this analysis but their results are not separately broken out from the IMS group. Future research could examine the subgroups within our three treated patient samples. Further, we do not claim that the costs, inpatient or otherwise, in the paper are uveitis-caused costs. Rather, we describe costs of patients with uveitis who are on three different treatments. Accordingly, these costs could be due to unrelated reasons, potentially related to uveitis, or directly related to uveitis. Identifying the causality for cost types is a highly challenging research problem and not something we set out to do. Nevertheless, these data are appropriate for studying all-cause costs and utilization, as they contain diagnosis, testing, and procedure information, and represent payments paid by insurers to providers, and not charges, for these patients. Finally, claims data are available for patients with private insurance entering the healthcare system for treatment and thus capture only those individuals who have used the health system and had a claim for services.

\section{Conclusions}

This analysis suggests that the burden of uveitis in the US privately insured population is economically significant. Patients treated with IMS or BIO were associated with improvements in rates of ophthalmologic comorbidities as well as reductions in non-study drug costs. Patients treated with IMS or BIO were also associated with improvements in rates of hospital admission and ER visits relative to CTS, which was associated with increases in these measures. Given the increase in comorbidity burden associated with CTS patients in the baseline and study comparison, there may be evidence that these patients are not appropriately treated.

\section{Additional file}

Additional file 1: Technical appendices.

\section{Abbreviations}

BIO: Biologics; CTS: Corticosteroids; ER: Emergency room; IMS: Immunosuppressants; PMPM: Per-member-per-month.

\section{Competing interests}

Chu has received honorarium from Analysis Group, Inc. which has received research funds from Novartis Pharmaceuticals Corporation. Johnson, Davis, Sorg, and Duh are employees of Analysis Group, Inc. Mallya is an employee of Novartis Pharmaceuticals Corporation. 


\section{Authors' contributions}

$D C, S J, U M, M D, R S$, and MSD designed the study. SJ, MD, RS, and MSD managed and analyzed the data. DC, SJ, MD, RS, and MSD interpreted the data and prepared the manuscript. All authors reviewed and approved the final manuscript.

\section{Acknowledgements}

Novartis Pharmaceuticals Corporation, East Hanover, NJ, USA, funded this research. The funding organization participated in the design of the study, including reviewing selection criteria and sensitivity analysis, and review of the manuscript.

\section{Author details \\ ${ }^{1}$ Institute of Ophthalmology and Visual Science, New Jersey Medical School, Rutgers University, 90 Bergen Street, Suite 6100, Newark, NJ 07960, USA. \\ ${ }^{2}$ Metropolitan Eye Research and Surgery Institute, Palisades Park, NJ, USA. \\ ${ }^{3}$ Analysis Group, Inc, 111 Huntington Avenue \#10, Boston, MA 02199, USA. \\ ${ }^{4}$ Novartis Pharmaceuticals Corporation, 1 Health Plaza, East Hanover, NJ 07936, USA.}

Received: 16 July 2013 Accepted: 7 October 2013

Published: 6 November 2013

\section{References}

1. Chang JH, Wakefield D (2002) Uveitis: a global perspective. Ocul Immunol Inflamm 10:263-279

2. Suttorp-Schulten MS, Rothova A (1996) The possible impact of uveitis in blindness: a literature survey. Br J Ophthalmol 80:844-848, doi:10.1136/ bjo.80.9.844

3. Darrell RW, Wagener HP, Kurland LT (1962) Epidemiology of uveitis: incidence and prevalence in a small urban community. Arch Ophthalmol 68:502-514, doi:10.1001/archopht.1962.00960030506014

4. Nussenblatt RB (1990) The natural history of uveitis. Int Ophthalmol 14(5-6):303-308, doi:10.1007/BF00163549

5. Durrani OM, Tehrani NN, Marr JE, Moradi P, Stavrou P, Murray PI (2004) Degree, duration, and causes of visual loss in uveitis. Br J Ophthalmol 88:1159-1162, doi:10.1136/bjo.2003.037226

6. Durrani OM, Meads CA, Murray PI (2004) Uveitis: a potentially blinding disease. Ophthalmologica 218:223-236, doi:10.1159/000078612

7. Nguyen QD, Callanazn D, Dugel P, Godfrey DG, Goldstein DA, Wilensky JT (2006) Treating chronic noninfectious posterior segment uveitis: the impact of cumulative damage. Proceedings of an expert panel roundtable discussion. Retina 26(suppl):1-16

8. Jabs DA, Rosenbaum JT, Foster CS, Holland GN, Jaffe GJ, Louie JS, Nussenblatt RB, Stiehm ER, Tessler H, Van Gelder RN, Whitcup SM, Yocum D (2000) Guidelines for the use of immunosuppressive drugs in patients with ocular inflammatory disorders: recommendations of an expert panel. Am J Ophthalmol 130(4):492-513

9. Stanbury RM, Graham EM (1998) Systemic corticosteroid therapy-side effects and their management. Br J Ophthalmol 82(6):704-708

10. Nguyen QD, Hatef E, Kayen B, Macahilig CP, Ibrahim M, Wang J, Shaikh O, Bodaghi B (2011) A cross-sectional study of the current treatment patterns in noninfectious uveitis among specialists in the United States. Ophthalmology 118(1):184-190, doi:10.1016/j.ophtha.2010.03.029

11. Imrie FR, Dick AD (2007) Nonsteroidal drugs for the treatment of noninfectious posterior and intermediate uveitis. Curr Opin Ophthalmol 18:212-219

12. Imrie FR, Dick AD (2007) Biologics in the treatment of uveitis. Curr Opin Ophthalmol 18:481-486

13. Gritz DC, Wong IG (2004) Incidence and prevalence of uveitis in Northern California. The Northern California Epidemiology of Uveitis Study. Ophthalmology 111:491-500

14. Reeves SW, Sloan FA, Lee PP, Jaffe GJ (2006) Uveitis in the elderly: epidemiological data from the National Long-term Care Survey Medicare Cohort. Ophthalmology 113:302-307

15. Schneeweiss S, Avorn JA (2005) Review of uses of health care utilization databases for epidemiologic research on therapeutics. J Clin Epi 58:323-337

16. Rein DB, Zhang P, Wirth KE, Lee PP, Hoerger TJ, McCall N, Klein R, Tielsch JM, Vijan S, Saaddine J (2006) The economic burden of major adult visual disorders in the United States. Arch Ophthalmol 124:1754-1760
17. Hodge WG (2004) Incidence and prevalence of uveitis in Northern California: discussion by. Ophthalmology 111:500, doi:10.1016/j. ophtha.2003.10.017

18. Deyo RA, Cherkin DC, Ciol MA (1992) Adapting a clinical comorbidity index for use with ICD-9-CM administrative databases. J Clin Epidemiol 45(6):613-619

19. Peterson AM, Nau DP, Cramer JA, Benner J, Gwadry-Sridhar F, Nichol M (2007) A checklist for medication compliance and persistence studies using retrospective databases. Value Health 10(1):3-12

20. McCannel CA, Holland GN, Helm CJ, Cornell PJ, Winston JV, Rimmer TG (1996) Causes of uveitis in the general practice of ophthalmology: UCLA Community-Based Uveitis Study Group. Am J Ophthalmol 121:35-46

21. Bundorf MK, Royalty A, Baker LC (2009) Health care cost growth among the privately insured. Health Aff (Millwood) 28(5):1294-1304, Costs inflation adjusted to 2009 dollars using the medical Consumer Price Index. doi:10.1377/hlthaff.28.5.1294

22. Laliberté F, Bookhart BK, Vekeman F, Corral M, Duh MS, Bailey RA, Piech CT, Lefebvre P (2009) Direct all-cause health care costs associated with chronic kidney disease in patients with diabetes and hypertension: a managed care perspective. J Manag Care Pharm 15(4):312-322, Costs inflation adjusted to 2009 dollars using the medical Consumer Price Index

23. Chang S, Long SR, Kutikova L, Bowman L, Finley D, Crown WH, Bennett CL (2004) Estimating the cost of cancer: results on the basis of claims data analyses for cancer patients diagnosed with seven types of cancer during 1999 to 2000. J Clin Oncol 22(17):3524-3530, doi:10.1200/JCO.2004.10.170, Costs inflation adjusted to 2009 dollars using the medical Consumer Price Index

24. Standardization of Uveitis Nomenclature (SUN) Working Group (2005) Standardization of uveitis nomenclature for reporting clinical data: results of the first international workshop. Am J Ophthalmol 140:509-516, doi:10.1016/j.ajo.2005.03.057

25. Epstein AM, Cumella EJ (1988) Capitation payment: using predictors for medical utilization to adjust rates. Health Care Financ Rev 10:51-69

26. Whittle J, Steinberg EP, Anderson GF, Herbert R (1991) Accuracy of Medicare claims data for estimation of cancer incidence and resection rates among elderly Americans. Med Care 29:1226-1236

doi:10.1186/1869-5760-3-64

Cite this article as: Chu et al:: Healthcare costs and utilization for privately insured patients treated for non-infectious uveitis in the USA. Journal of Ophthalmic Inflammation and Infection 2013 3:64.

\section{Submit your manuscript to a SpringerOpen ${ }^{\circ}$ journal and benefit from:}

- Convenient online submission

- Rigorous peer review

- Immediate publication on acceptance

- Open access: articles freely available online

- High visibility within the field

- Retaining the copyright to your article

Submit your next manuscript at $>$ springeropen.com 